\title{
PROPUESTA DE UN SISTEMA DE GESTIÓN DE LA CONTAMINACIÓN SONORA EN LA CIUDAD DE ANDAHUAYLAS, APURÍMAC, 2016
}

\author{
PROPOSAL OF A SOUND POLLUTION MANAGEMENT SYSTEM \\ IN THE CITY OF ANDAHUAYLAS, APURIMAC, 2016
}

\author{
Julio C. Sichez Muñoz ${ }^{1}$
}

\section{RESUMEN}

El ruido o contaminación sonora, es considerado el flagelo del presente siglo, es por ello que en el presente estudio se planteó como objetivo general; proponer un Sistema de Gestión de la Contaminación Sonora a partir de la relación entre Contaminación Sonora y Nivel de Ansiedad de la Población en la Ciudad de Andahuaylas, Región Apurímac, 2016; la metodología utilizada consistió en identificar las zonas de muestreo para medir el nivel de presión sonora (ruido) y el nivel de ansiedad de la población de la ciudad de Andahuaylas. Se determinó que existe una correlación moderada positiva significativa entre ansiedad y ruido $(r=0,258)$ $(p=0,011)$, es decir, a mayor intensidad de ruido, moderadamente incrementa la ansiedad en los pobladores de la ciudad de Andahuaylas. Ante la prueba de Chi cuadrado, existe relación significativa entre los niveles de ansiedad y ruido $(\mathrm{p}=0,000)$ en los pobladores de la ciudad de Andahuaylas. Ante la prueba de riesgo, la exposición a regular ruido se constituye en un riesgo muy elevado para una ansiedad moderada $(\mathrm{OR}=6,2)$, es decir existe 6.2 veces más riesgo que los pobladores presenten ansiedad moderada si están expuesto a regular ruido, que a bajo ruido. Considerando que existe una correlación moderada se ha planteado un sistema de gestión de la Contaminación Sonora para la ciudad de Andahuaylas.

PALABRAS CLAVE: Nivel de presión sonora; nivel de ansiedad; Sistema de Gestión

1 Maestro en Ciencias, mención Gestión Ambiental, Universidad Nacional José María Arguedas. Docente de la Universidad Nacional José María Arguedas, Facultad de Ingeniería, Escuela Profesional de Ingeniería Agroindustrial, Andahuaylas, Apurímac, Perú. 


\section{ABSTRACT}

The noise or noise pollution is considered the scourge of the present century, that is why in the present study it was proposed as a General Objective; To propose a Sound Pollution Management System based on the relationship between Sound Pollution and Population Anxiety Level in the City of Andahuaylas, Apurímac Region, 2016; The methodology used consisted in identifying the sampling zones to measure the level of sound pressure (noise) and the level of anxiety of the population of the city of Andahuaylas. It was determined that there is a moderate positive significant correlation between Anxiety and Noise $(r=0.258)$ $(p=0.011)$, that is, at higher noise intensity, moderately increases anxiety in the inhabitants of the city of Andahuaylas. Before the Chi square test, there is a significant relationship between levels of anxiety and noise $(\mathrm{p}=0.000)$ in the inhabitants of the city of Andahuaylas. Exposure to regular noise is a very high risk for moderate anxiety $(O R=6.2)$, that is to say, there is a 6.2 times greater risk that the residents present moderate anxiety if they are ex-posed to regular noise, Which at low noise. Considering that there is a moderate correlation, a sound pollution management system has been proposed for the city of Andahuaylas

KEY WORDS: Sound pressure level; Anxiety level; Management system.

\section{INTRODUCCIÓN}

La ciudad de Andahuaylas es el núcleo urbano más importante de la provincia de Andahuaylas, de la región Apurímac, por el valioso desarrollo económico y de las diferentes actividades cívicas, socioculturales y económicas que en ella se realizan. Sin embargo, estas actividades sumado a la falta de planificación del tránsito vehicular, entre otras actividades no suelen ser las mejores desde el punto de vista de la contaminación sonora y sus impactos en la población; más aún si consideramos que el ruido pone en riesgo la salud de la población por sus efectos negativos que tiene en ella ${ }^{1}$.

En el trabajo de Investigación, "Contaminación sonora e impactos en la población de Chimbote 1999”, se concluyó que existe relación significativa entre la contaminación sonora y el bienestar de la población de Chimbote y que los niveles de contaminación sonora superaban los límites máximos permisibles².

En el trabajo de investigación, "Impactos ambientales y fuentes de contaminación ambiental de la provincia de Andahuaylas de la región Apurímac. 2013 2014", se concluye que las principales fuentes de contaminación ambiental de la Provincia de Andahuaylas, Región Apurímac son: los desechos sólidos municipales, el parque automotor de la provincia, el Botadero Municipal de Desechos Sólidos Municipales de Andahuaylas y la Contaminación Sonora ${ }^{1}$. 
Se considera que el ruido es un sonido no deseado. Para comprender mejor el significado del concepto ruido se consideró los aportes de las distintas disciplinas, una de ellas es la Ciencia de la Comunicación que agrupa bajo el nombre de ruido a todas las molestias y obstáculos a la comunicación de origen exterior (ruido de fondo, interferencias, etc. $)^{3}$.

El ruido, considerado como agente contaminante ambiental, posee características que pueden causar daños o molestias a las personas. Sin embargo es necesario resaltar que los sonidos, como tal, están relacionados con casi la totalidad de las actividades humanas. Considerando la concepción subjetiva que se le da al ruido, se debe considerar que no todos los ruidos son molestos o perjudiciales, sin embargo, cuando se juntan en un momento determinado estos pueden ser perjudiciales para la persona. En las ciudades el mayor aporte corresponde a "fuentes móviles", es decir, los autos, ómnibus, motos, camiones, etc. Esto es lo que se define como Ruido Urbano ${ }^{3}$.

En la Pontificia Universidad Católica del Perú los niveles o intensidades de ruido son superiores a los recomendados para las actividades académicas universitarias dentro del campus según recomendaciones de instituciones nacionales e internacionales y que los mapas de ruido muestran una tendencia cíclica. La principal fuente proviene del sector transporte, específicamente de los vehículos que transitan por la Av. Universitaria y Riva Agüero ${ }^{4}$.

Los principales efectos de la contaminación sonora (ruido) en las personas, se dan en los Efectos del ruido sobre la audición: en la Anatomía y fisiología del oído humano y las pérdidas de capacidad auditiva; Efectos mediados por la reacción de estrés; Efectos sobre el aparato cardiovascular; Interferencia con la comunicación; Interferencia con la realización de tareas; Interferencia del ruido con el sueño; Molestia subjetiva; Efectos del ruido sobre la salud mental y Efectos económicos del ruido 5 .

El alto número de vehículos que componen el parque automotriz de la ciudad de Puerto Montt en Chile, es el principal agente contamínate de ruido en la zona evaluada, a esto sumamos los malos hábitos de conducción que demuestran los conductores, tales como, exceso de velocidad, silenciadores en mal estado o modificados, el exceso de uso de bocinas, etc. La variabilidad de niveles, muestran niveles promedios en periodo diurno entorno a los $65 \mathrm{~dB}(\mathrm{~A})$ y en periodo nocturno entorno a los $50 \mathrm{~dB}(\mathrm{~A})$, esto en días de semana ${ }^{5}$.

El tráfico rodado es, con gran diferencia, la principal fuente de ruido ambiental en algunos tramos de la ciudad de Valencia. El análisis de los mapas de 
ruido obtenidos ha revelado que los niveles sonoros equivalentes encontrados, cumplen con los límites establecidos en la legislación vigente en zonas de uso industrial, es decir; por debajo de los $70 \mathrm{~dB}$ (A) para el periodo diurno e inferiores a los $60 \mathrm{~dB}(\mathrm{~A})$ para el período nocturno ${ }^{6}$.

El nivel de contaminación sonora (ruido) en el centro de la ciudad de Huacho se encuentra entre 65 a $85 \mathrm{~dB}(\mathrm{~A})$, específicamente están por encima de $66 \mathrm{~dB}(\mathrm{~A})$, y respecto al nivel de estrés de los pobladores el 73,10\% está en el nivel de estrés moderado. De igual forma concluyó que de acuerdo a la percepción de la población en estudio la causa principal del ruido es el tránsito vehicular $(84,9 \%)^{7}$.

La implementación del sistema de gestión ambiental en los municipios busca fortalecer la capacidad de gestión ambiental municipal, para consolidar la condición del municipio como eje natural de coordinación entre entidades públicas y los actores sociales en el nivel local elevando el compromiso, derechos, deberes y la participación de la administración municipal y los diferentes actores sociales en la gestión ambiental para que se fortalezca su capacidad institucional en los temas ambientales que le compete.

La Intensidad de ruido promedio general registrado en el Centro Urbano de la Ciudad de Tarapoto fue de 83,3 dB, un 28,2\% más alto que el Límite Tolerable establecido por la Organización Mundial de la Salud (OMS) ${ }^{9}$.

Algunas fuentes fijas que podemos encontrar en la ciudad de Valdivia (Chile) son las provenientes de las industrias, de los colegios, de los locales públicos, las viviendas, ferias libres establecidas, del sector construcción. Respecto a algunas fuentes móviles que podemos encontrar en la ciudad son las provenientes del tráfico vehicular, del tráfico ferroviario, del tráfico aéreo ${ }^{10}$.

La Gestión Ambiental del ruido incluye todas las actuaciones que tienen por objeto prevenir o reducir la contaminación acústica a la que está expuesta la población, y la preservación y mejora de la calidad acústica del territorio. Las acciones pueden ser tanto de corrección como de prevención. Por otra parte, la gestión ambiental del ruido debe prever la incorporación de criterios acústicos en el planeamiento para hacer compatibles los distintos usos en el territorio ${ }^{11}$.

El Objetivo General del estudio fue; Proponer un Sistema de Gestión de la Contaminación Sonora a partir de la relación entre Contaminación Sonora y Nivel de Ansiedad de la Población en la Ciudad de Andahuaylas, Región Apurímac, 2016. 
Los Objetivos Específicos que se plantearon fueron:

1) Identificar el nivel de contaminación sonora de la ciudad de Andahuaylas, Región Apurímac. 2016.

2) Determinar el nivel de ansiedad de la población expuesta a contaminación sonora en la ciudad de Andahuaylas, Región Apurímac. 2016.

3) Determinar la relación entre el nivel de contaminación sonora y ansiedad de la población expuesta a contaminación sonora en la ciudad de Andahuaylas, Región Apurímac 2016.

4) Proponer un Sistema de Gestión de la Contaminación Sonora en la ciudad de Andahuaylas, Región Apurímac. 2016.

\section{MATERIALES Y MÉTODOS}

\section{POBLACIÓN, MUESTRA Y VARIABLES}

El material de estudio lo constituyó la población de la ciudad de Andahuaylas, de la provincia de Andahuaylas región Apurímac, expuesta a la contaminación sonora, las que fueron previamente seleccionadas.

\section{Variables de estudio}

V1: Nivel de Contaminación

V2: Nivel de Ansiedad

\section{DISEÑO}

La investigación es No Experimental, descriptiva correlacional, porque examina la relación o asociación existente entre las dos variables de estudio, en la misma unidad de investigación o sujetos de estudio y se propone un Plan de Gestión para la Contaminación Sonora de la ciudad de Andahuaylas.

\section{Metodología}

La metodología utilizada fue la siguiente:

- Se determinó las zonas de muestreo para medir la intensidad de ruido, mediante el uso del sonómetro.

- Se seleccionó la muestra de estudio de acuerdo a las zonas de monitoreo.

- La medición del NPS se realizó en diferentes horas, y las mediciones se tomaron cada 10 segundos. 
- Determinada la muestra de estudio se aplicó el test de Zunt y del cuestionario de preguntas.

- Recogido los datos del estudio, se tabularon estos en tablas estadísticos.

- Se realizó el análisis estadístico de los resultados obtenidos.

\section{RESULTADOS}

Tabla 1

NIVEL DE PRESIÓN SONORA DE LAS ZONAS DE MONITOREO.

ANDAHUAYLAS. 2016

\begin{tabular}{|c|c|c|c|c|c|c|}
\hline \multirow{2}{*}{$\begin{array}{l}\text { Zona de } \\
\text { monitoreo }\end{array}$} & \multicolumn{6}{|c|}{ Nivel de presión sonora $(\mathrm{dB})$} \\
\hline & 1 & 2 & 3 & 4 & 5 & 6 \\
\hline 1 & 68 & 60 & 63 & 61 & 70 & 65 \\
\hline 2 & 63 & 60 & 65 & 59 & 70 & 64 \\
\hline 3 & 70 & 67 & 71 & 70 & 74 & 69 \\
\hline 4 & 70 & 68 & 71 & 74 & 72 & 68 \\
\hline 5 & 72 & 69 & 73 & 76 & 75 & 81 \\
\hline 6 & 69 & 58 & 57 & 56 & 53 & 72 \\
\hline 7 & 57 & 65 & 70 & 69 & 67 & 71 \\
\hline 8 & 71 & 80 & 82 & 84 & 74 & 80 \\
\hline 9 & 51 & 53 & 59 & 55 & 57 & 50 \\
\hline 10 & 63 & 61 & 64 & 64 & 67 & 65 \\
\hline 11 & 70 & 68 & 62 & 66 & 74 & 70 \\
\hline 12 & 73 & 70 & 68 & 60 & 56 & 57 \\
\hline 13 & 57 & 58 & 57 & 60 & 55 & 69 \\
\hline 14 & 54 & 53 & 52 & 66 & 69 & 72 \\
\hline 15 & 62 & 69 & 66 & 69 & 70 & 70 \\
\hline 16 & 70 & 67 & 75 & 79 & 69 & 70 \\
\hline 17 & 74 & 70 & 73 & 72 & 79 & 70 \\
\hline 18 & 69 & 71 & 70 & 67 & 66 & 77 \\
\hline 19 & 76 & 75 & 67 & 71 & 69 & 68 \\
\hline 20 & 76 & 72 & 66 & 65 & 63 & 62 \\
\hline 21 & 76 & 75 & 77 & 72 & 75 & 73 \\
\hline 22 & 81 & 76 & 80 & 79 & 80 & 78 \\
\hline 23 & 67 & 71 & 68 & 70 & 72 & 72 \\
\hline 24 & 72 & 71 & 70 & 72 & 76 & 75 \\
\hline
\end{tabular}


Tabla 2

NIVEL DE ANSIEDAD DE LOS POBLADORES DE LA CIUDAD DE ANDAHUAYLAS DE ACUERDO A LAS ZONAS DE MONITOREO. 2016

\begin{tabular}{|c|c|c|c|c|}
\hline \multirow{2}{*}{$\begin{array}{c}\begin{array}{c}\text { Zona de } \\
\text { monitoreo }\end{array} \\
1\end{array}$} & \multirow[b]{2}{*}{40} & \multicolumn{2}{|c|}{ Nivel de Ansiedad } & \multirow[b]{2}{*}{39} \\
\hline & & 42 & 59 & \\
\hline 2 & 58 & 59 & 58 & 60 \\
\hline 3 & 66 & 64 & 50 & 60 \\
\hline 4 & 48 & 46 & 52 & 48 \\
\hline 5 & 55 & 54 & 64 & 55 \\
\hline 6 & 60 & 62 & 64 & 63 \\
\hline 7 & 66 & 65 & 60 & 67 \\
\hline 8 & 58 & 62 & 69 & 59 \\
\hline 9 & 72 & 70 & 59 & 70 \\
\hline 10 & 58 & 60 & 50 & 64 \\
\hline 11 & 59 & 58 & 60 & 54 \\
\hline 12 & 56 & 54 & 55 & 50 \\
\hline 13 & 62 & 60 & 64 & 65 \\
\hline 14 & 45 & 40 & 38 & 41 \\
\hline 15 & 64 & 66 & 68 & 66 \\
\hline 16 & 58 & 56 & 57 & 54 \\
\hline 17 & 66 & 68 & 70 & 64 \\
\hline 18 & 50 & 54 & 52 & 56 \\
\hline 19 & 74 & 72 & 70 & 74 \\
\hline 20 & 79 & 78 & 74 & 76 \\
\hline 21 & 70 & 72 & 70 & 66 \\
\hline 22 & 79 & 75 & 78 & 70 \\
\hline 23 & 70 & 75 & 68 & 66 \\
\hline 24 & 76 & 70 & 68 & 72 \\
\hline
\end{tabular}


Julio C. Sichez Muñoz

Tabla 3

DISTRIBUCIÓN DE LA POBLACIÓN ENCUESTADA

DE ACUERDO A LA EDAD

\begin{tabular}{lcc}
\hline Rango de Edad (años) & Fi & hi $\%$ \\
\hline Menos 18 años & 14 & 14,58 \\
Entre 18 y 35 años & 44 & 45,83 \\
Entre 36 y 50 años & 32 & 33,33 \\
Más de 50 años & 6 & 6,25 \\
\hline Total & 96 & 100,00 \\
\hline
\end{tabular}

Tabla 4

CUANDO VIAJA EN SERVICIO PÚBLICO DE TRANSPORTE

EL CONDUCTOR UTILIZA ADECUADAMENTE

EL CLAXON DEL VEHÍCULO

\begin{tabular}{ccc}
\hline Respuesta & Fi & hi \% \\
\hline Si & 27 & 28,13 \\
No & 69 & 71,88 \\
\hline Total & 96 & 100,00 \\
\hline
\end{tabular}

Tabla 5

CUAL ES SU OPINIÓN SOBRE CONTAMINACIÓN SONORA

(RUIDO)

\begin{tabular}{lcc}
\hline Respuesta & Fi & hi $\%$ \\
\hline Molesta, fastidia & 31 & 32,29 \\
Afecta nuestra salud & 26 & 27,08 \\
Nos pone nerviosos, ansioso & 39 & 40,63 \\
\hline Total & 96 & 100,00 \\
\hline
\end{tabular}


Tabla 6

CUAL ES LA PRINCIPAL CAUSA DE LA CONTAMINACIÓN SONORA EN ANDAHUAYLAS

\begin{tabular}{lcc}
\hline Respuesta & Fi & hi \% \\
\hline Tránsito vehicular & 19 & 19,79 \\
Uso excesivo claxon por choferes & 39 & 40,63 \\
Presencia de Peñas y Discotecas & 6 & 6,25 \\
Las Moto taxis & 32 & 33,33 \\
\hline Total & 96 & 100,00 \\
\hline
\end{tabular}

Tabla 7

DE ACUERDO A SU PERCEPCIÓN. QUE RUIDO LE MOLESTA MÁS

\begin{tabular}{lcc}
\hline Respuesta & Fi & hi $\%$ \\
\hline Vehículos & 68 & 70.83 \\
Discotecas & 14 & 14.58 \\
Vecinos & 6 & 6.25 \\
Policía Transito & 8 & 8.33 \\
\hline Total & 96 & 100,00 \\
\hline
\end{tabular}

Tabla 8

ALGUNA RECOMENDACIÓN ANTE EL PROBLEMA DEL RUIDO

\begin{tabular}{lcc}
\hline Respuesta & Fi & hi \% \\
\hline Se apliquen leyes del Alcalde para el ruido & 15 & 15.63 \\
Se prohíba uso del claxon & 42 & 43.75 \\
Se reacomode el tránsito vehicular & 33 & 34.38 \\
Se eliminen las peñas, discotecas de la ciudad & 6 & 6.25 \\
\hline Total & 96 & 100,00 \\
\hline
\end{tabular}


En la tabla 1 se muestran los resultados promedios del Nivel de Presión Sonora, tomados en las 24 zonas de monitoreo de la ciudad de Andahuaylas. En la tabla 2, se presentan los resultados de los niveles de ansiedad de los pobladores de la ciudad de Andahuaylas. Respecto a las opiniones de los pobladores de la ciudad de Andahuaylas, sobre la problemática de la contaminación sonora (ruido), se tuvieron resultados que nos ha permitido tomas decisiones respecto a las instituciones que deben de participar en el Sistema de Gestión de la Contaminación Sonora que se propone para Andahuaylas.

De acuerdo a la tabla 3 , se tiene que el $45,83 \%$ de la población de estudio tenían entre 18 y 35 años, el 33,33\% entre 36 y 50 años, el 14,58\% menor a 18 años y solo el 6,25\% más de 50 años. Con respecto al sexo se tuvo que el 60,42\% eran de sexo masculino y el $39,58 \%$ de sexo femenino. Respecto, si consideraba que el ruido de su sector le molesta para poder vivir, se obtuvo que para el 73,96\% si le molesta y el $26,04 \%$ no le molestaba. Otro dato importante, fue respecto a que si el ruido afectaba su salud, la población respondió que SI en un 76,04\% y que $\mathrm{NO}$ el 23,96\%. Respecto a conocer si el conductor de transporte publico utiliza adecuadamente el claxon del vehículo que conduce, el 71,88\% respondió que $\mathrm{NO}$ utilizaba adecuadamente el claxon del vehículo en tanto que el 28,13\% respondió que SI lo hacía adecuadamente (Tabla 4).

Respecto a la opinión de cada persona sobre Contaminación Sonora (ruido), el 40,63\% respondió que los ponía nerviosos o ansiosos, al 32,29\% les molesta o fastidia y al 27,08\% les afecta la salud (Tabla 5). Respecto a que si consideraba que el ruido influye en la calidad de vida de las personas, para el 73,96\% SI influye, en tanto que para el 26,04\% NO influía en la calidad de vida. Respecto a determinar cuál es la causa principal de la contaminación sonora en la ciudad de Andahuaylas (Tabla 6), el 40,63\% respondió que el uso excesivo del claxon por choferes era la principal causa del ruido, el 33,33\% eran los moto taxis, el 19,79\% era el tránsito vehicular y el 6,25\% que era la presencia de peñas y discotecas.

Respecto a la pregunta, los datos se muestran en la tabla 7, de acuerdo a su percepción que ruido le molesta más, se tuvo que el 70,83\% opina que era el ruido de los vehículos, el 14,58\% el ruido producido por las discotecas, el 8,33\% el ruido producido por la policía de tránsito y el 6,25\% el ruido producido por los vecinos. En la tabla 08, se presentan los resultados, respecto a alguna recomendación que podrían dar ante el problema del ruido en la ciudad de Andahuaylas, se tuvo que para el $43,75 \%$ se debería de prohibir el uso del claxon de parte de los 
choferes, el 34,38\% que se debería reacomodar el transito vehiculas, el 15,63\% que se deberían de aplicar leyes de Alcaldía para el ruido y el 6,25\% que se eliminen las peñas y discotecas de la ciudad.

\section{DISCUSIÓN}

De acuerdo a los resultados obtenidos se tiene que el máximo puntaje obtenido para el Nivel de Ansiedad es 66 puntos, y el mínimo 38, con un promedio de $57,9 \pm 5,96$ puntos. El nivel de ansiedad que predomina es la ansiedad moderada $(66,7 \%)$ seguido de la ansiedad severa $(28,1 \%)$ y con mínima proporción sin ansiedad $(5,2 \%)$ en los pobladores de la ciudad de Andahuaylas. Respecto al Nivel de Contaminación Sonora se tiene que el máximo de NPS (dB) obtenido es de 76,6 dB, y el mínimo 60,4 dB, con un promedio de 69,09 44,18 dB. El nivel de ruido que prevalece es de regular ruido $(83,3 \%)$ seguido con menor proporción bajo ruido $(16,7 \%)$ en la ciudad de Andahuaylas.

Al aplicar la Prueba de Correlación de Pearson entre Ansiedad y Ruido, se puede explicar que, existe una correlación moderada positiva significativa entre Ansiedad y Ruido $(\mathrm{r}=0,258)(\mathrm{p}=0,011)$, es decir, a mayor intensidad de ruido, moderadamente incrementa la ansiedad en los pobladores de la ciudad de Andahuaylas. Respecto a la Prueba No Paramétrica (Chi-Cuadrado de Pearson), para determinar la Relación o Asociación entre Niveles de Ansiedad y Ruido, se determinó que entre los que están expuesto a bajo ruido, el 43,8\% presenta ansiedad severa, el 31,3\% ansiedad moderada y el 25\% sin ansiedad. A diferencia de la población expuesta a Regular ruido, donde prevalece la ansiedad moderada $(73,8 \%)$, seguido con menor proporción ansiedad severa $(25,0 \%)$ y solo el $1,3 \%$ sin ansiedad. Ante la prueba de Chi cuadrado, existe relación significativa entre los niveles de ansiedad y ruido $(\mathrm{p}=0,000)$ en los pobladores de la ciudad de Andahuaylas.

Respecto a la Prueba Epidemiológica de Riesgo (Odd Ratio - Or), para determinar el Grado de Exposición sobre el Daño, entre la Exposición (Regular ruido) y el Daño (Ansiedad moderada), se tuvo que, la exposición a regular ruido se constituye en un riesgo muy elevado para una ansiedad moderada $(O R=6,2)$, es decir existe 6,2 veces más riesgo que los pobladores presenten ansiedad moderada si están expuesto a regular ruido, que a bajo ruido.

Estos resultados se asemejan a los encontrados por Rodríguez, Y. y otros en su trabajo de Contaminación sonora e impactos en la población de Chimbote 
1999, que encontraron que existía correlación significativa entre ruido y nivel de ansiedad; así mismo Sichez, J. y otros determinaron que la Contaminación Sonora en la ciudad de Andahuaylas era uno de los factores de incidencia en los niveles de contaminación ambiental de la provincia de Andahuaylas. De igual forma estos resultados coinciden con los obtenidos por Baca y Seminario quien sostenía que la fuente principal del ruido en la Pontificia Universidad Católica del Perú eran los provenientes de los vehículos que transitan la Av. Universitaria y Riva Agüero.

En conclusión; Existe una correlación moderada positiva significativa entre Ansiedad y Ruido $(\mathrm{r}=0,258)(\mathrm{p}=0,011)$, es decir, a mayor intensidad de ruido, moderadamente incrementa la ansiedad en los pobladores de la ciudad de Andahuaylas.

\section{PROPUESTA DE UN SISTEMA DE GESTIÓN DE LA CONTAMINACIÓN SONORA EN LA CIUDAD DE ANDAHUAYLAS, APURÍMAC, 2016}

\section{POLÍTICA AMBIENTAL}

Mejoramiento del bienestar de la población de la ciudad de Andahuaylas, en base al desarrollo de un Sistema de Gestión de la Contaminación Sonora adecuado para la ciudad de Andahuaylas, buscando el desarrollo sostenible de la ciudad, con la participación de sus autoridades, representaciones de los Colegios Profesionales, instituciones públicas y privadas y de la comunidad, dentro del marco legal ambiental existente.

\section{OBJETIVOS}

\subsection{Objetivo General}

Organizar la gestión ambiental de la contaminación sonora de la ciudad de Andahuaylas, implementando el Comité de Gestión Ambiental Sonoro de la ciudad de Andahuaylas.

\subsection{Objetivos Específicos}

- Desarrollar el Sistema de gestión de la contaminación sonora de la ciudad de Andahuaylas, de acuerdo a la normativa legal existente. 
- Elaborar e implementar las normas municipales adecuadas que permitan un control adecuado de la contaminación sonora en la ciudad de Andahuaylas.

- Elaborar y ejecutar un Programa de Educación Ambiental, sobre contaminación sonora, a nivel universitario y de centros educativos secundarios y primarios, con el propósito de concientizar ambientalmente a los estudiantes de estos niveles educativos.

- Elaborar y proponer un Plan estratégico sobre tránsito vehicular de la ciudad de Andahuaylas, buscando el reordenamiento del sector.

- Elaborar y proponer un Plan estratégico sobre autorización de funcionamiento de centros de diversión nocturna de la ciudad de Andahuaylas, buscando el reordenamiento del sector.

- Desarrollar programas de monitoreo de niveles de contaminación sonora en diferentes zonas críticas de la ciudad de Andahuaylas, con el propósito de controlar la emisión de ruidos molestos que perjudiquen o afecten el bienestar de la población de Andahuaylas.

\section{METAS}

- Reglamentar con Ordenanzas Municipales, las actividades industriales y comerciales, así como las actividades de servicios, respecto a la emisión de ruidos, en un plazo no mayor de 02 años.

- Concientizar a la población estudiantil en aspectos sobre control de la contaminación sonora en la ciudad de Andahuaylas, desarrollando por lo menos 02 programas de capacitación en educación ambiental por año.

- Disminuir en los próximos 5 años, en un 40 \% la emisión de ruidos molestos en la ciudad de Andahuaylas.

\section{ORGANIZACIONES COMPROMETIDAS:}

Municipalidad Provincial de Andahuaylas, Gobierno Subregional Chanka, Comité Provincial de Defensa Civil, Dirección Regional de Salud Ambiental, Organismos No Gubernamentales, Colegios Profesionales de Andahuaylas y Universidades de la ciudad de Andahuaylas 


\section{AUTORIDAD COMPETENTE O RESPONSABLE}

Municipalidad Provincial de Andahuaylas.

\section{PROPUESTA DE ACTIVIDADES}

- Elaboración del Mapa de ruidos de la ciudad de Andahuaylas, mediante el monitoreo de los puntos críticos de la ciudad de Andahuaylas, respecto a la contaminación sonora

- Elaboración del Inventario de fuentes de emisión de contaminación sonora en la ciudad de Andahuaylas, mediante el análisis y seguimiento de las principales fuentes, que se determinarán en base a estudios de control respecto a la emisión de ruidos frecuentes

- Elaboración de ordenanzas municipales, orientadas a reglamentar la emisión de contaminación sonora en la ciudad de Andahuaylas, en base a los datos que se obtengan del monitoreo ambiental respecto al ruido.

- Elaboración y desarrollo de un Programa de salud ambiental.

- Elaboración y desarrollo de un programa de capacitación en Educación Ambiental Sonora, para los diferentes niveles de educación en Andahuaylas.

- Elaboración del Programa de planificación del tránsito vehicular en Andahuaylas.

\section{REFERENCIAS BIBLIOGRÁFICAS}

1. Sichez, J.; García, C.; Rojas, W. "Impactos ambientales y fuentes de contaminación ambiental de la provincia de Andahuaylas de la región Apurímac. 2013 - 2014”. Trabajo de Investigación, Universidad Nacional José María Arguedas. Apurímac. 2014

2. Rodríguez, Y.; Sichez, J.; Ponce, M.; Melgarejo, E. "Contaminación sonora e impactos en la población de Chimbote 1999”. Trabajo de Investigación 1999. Universidad Nacional del Santa. Ancash. 2000

3. Rendiles, H. "Efectos de la exposición industrial". Venezuela. edit. Maracaibo S.A. 3era.ed. pág. 122.2008

4. Baca, W.; Seminario, S. "Evaluación de impacto sonoro en la Pontificia Universidad Católica del Perú”, tesis para optar por el Título de Ingeniero Civil. 2012

5. Lobos, V. "Evaluación del ruido ambiental en la ciudad de Puerto Montt". Tesis presentada para optar al grado académico de Licenciado en Acústica y el Título Profesional de Ingeniero Acústico. Valdivia - Chile. 2008 
6. Aliaga, J.; Martínez, J. "Estudio acústico de impacto ambiental y cálculo de la incertidumbre asociada a los niveles sonoros del estado preoperacional en el Sector Industrial I-1 del municipio de Náquera". 2013

7. León, R. Tesis "Caracterización de la Contaminación Sonora y su Influencia en la Calidad de Vida en los Pobladores del Centro de la Ciudad de Huacho, 2010-2011”. Tesis para optar el Grado Académico de Maestro en Ecología y Gestión Ambiental. Huacho. Perú. 2012

8. Obando, A. "Estrategia para la Implementación del Sistema de Gestión Ambiental Municipal (SIGAM) en el Municipio de Quinchía Risaralda”. Tesis para Optar el Titulo de Administrador Ambiental. Universidad Tecnológica de Pereira. Colombia. 2014.

9. Vergara, S.; Torres, J.; Gamboa, M. "Contaminación Sonora en el Centro Urbano de la Ciudad de Tarapoto. 2004”. Tarapoto. 2004

10. González, S. "Elaboración de una Encuesta sobre Percepción de Ruido Ambiental para ser aplicadas en familias del Programa Puente de la Comuna de Chimbarongo". Tesis para optar el Grado de Licenciado en Acústica y el Título Profesional de Ingeniero Acústico. Universidad Austral de Chile. Valdivia. Chile. 2006

11. Vilca, H. "Gestión por Ruido Ambiental". Universidad Nacional de San Agustín. Escuela de Posgrado. Doctorado en Ciencias: Salud Pública. Arequipa. Perú. 2013

12. Organización Panamericana de la Salud. Criterios de salud ambiental: el ruido, publicación científica 164, edit. OPS, México. 1983. 